\title{
catalysts
}

ISSN 2073-4344

www.mdpi.com/journal/catalysts

Article

\section{Application of "Boomerang" Linear Polystyrene-Stabilized Pd Nanoparticles to a Series of C-C Coupling Reactions in Water}

\section{Atsushi Ohtaka ${ }^{1, *}$, Toshiyuki Okagaki ${ }^{1}$, Go Hamasaka ${ }^{2}$, Yasuhiro Uozumi ${ }^{2}$, Tsutomu Shinagawa ${ }^{3}$, Osamu Shimomura ${ }^{1}$ and Ryôki Nomura ${ }^{1,4}$}

1 Department of Applied Chemistry, Faculty of Engineering, Osaka Institute of Technology, 5-16-1 Ohmiya, Asahi, Osaka 535-8585, Japan; E-Mails: m1m13504@st.oit.ac.jp (T.O.); shimomura@chem.oit.ac.jp (O.S.); nomura@chem.oit.ac.jp (R.N.)

2 Institute for Molecular Science (IMS), Higashiyama 5-1, Myodaiji, Okazaki 444-8787, Japan; E-Mails: hamasaka@ims.ac.jp (G.H.); uo@ims.ac.jp (Y.U.)

3 Osaka Municipal Technical Research Institute, 1-6-50 Morinomiya, Joto, Osaka, 536-8553, Japan; E-Mail: tshina@omtri.or.jp

4 Nanomaterials and Microdevices Research Center, Osaka Institute of Technology, 5-16-1 Ohmiya, Asahi, Osaka 535-8585, Japan

* Author to whom correspondence should be addressed; E-Mail: otaka@chem.oit.ac.jp; Tel.: +81-6-6954-4729; Fax: +81-6-6957-2135.

Academic Editors: Masahiko Arai and Fengyu Zhao

Received: 19 December 2014 / Accepted: 3 February 2015 / Published: 9 February 2015

\begin{abstract}
The application of a catch-and-release system for soluble Pd species between water (reaction medium) and polystyrene (polymer support) was examined in the Suzuki coupling reaction with 2-bromothiophene and the Heck reaction with styrene or bromobenzene. Although a slight increase in particle size was observed by TEM after re-stabilization of the Pd species on linear polystyrene, no agglomeration was observed.
\end{abstract}

Keywords: nanoparticles; boomerang catalyst; C-C coupling; water 


\section{Introduction}

Recently, homogeneous-heterogeneous switching catalysts have become of great interest to chemists, because they have the advantages of both homogeneous (high activity) and heterogeneous (recovery and recyclability) catalysts [1-4]. For example, Ikegami and Aoshima have developed homogeneous-heterogeneous switching catalysts by using thermosensitive polymers, such as poly( $N$-isopropylacrylamide) and poly[2-(2-ethoxy)ethoxyethyl vinyl ethers]. We have also reported a switching catalyst using a $\mathrm{pH}$-sensitive polyion complex. A polymer supported "boomerang" catalyst that operates through the reversible release of a homogeneous catalyst into the solution phase from the polymer support is another example of a homogeneous-heterogeneous switching catalyst [5-10]. For instance, Reiser et al. have developed a method for the reversible immobilization of pyrene-tagged palladium $N$-heterocyclic carbene (NHC) complexes on highly magnetic, graphene-coated cobalt nanoparticles through $\pi-\pi$ stacking interactions.

On the other hand, metal nanoparticles possess high catalytic activity in water [11-19] and have been used not only as semi-heterogeneous catalysts, but also as semi-heterogeneous supports [20-23]. Unfortunately, heterogenization of metal nanoparticles decreases catalytic activity. Additionally, the recovery and reuse of metal nanoparticles have been difficult to achieve due to a significant loss and/or morphology changes of the metal nanoparticles during reaction and workup [13-15]. Since leaching of soluble metal species from the support is a major cause of catalyst deactivation, efforts have been reported to develop an effective support that prevents leaching of metal species [16-19]. In contrast, leaching of $\mathrm{Pd}$ species has been observed directly and shows high catalytic activity in some systems [24-26]. Recently, palladium nanoparticles generated in situ for the Suzuki coupling reaction and aerobic alcohol oxidation in water were recovered using linear polystyrene, even in the presence of organic compounds [27]. These reports prompted efforts to develop recyclable "boomerang" nanoparticles that are able to overcome the problems mentioned above. Previously, we developed a catch-and-release system for soluble Pd species in water using linear polystyrene as an efficient reservoir [28]. This report describes the application of this system to a series of coupling reactions.

\section{Results and Discussion}

In the previous report, we confirmed that a recyclable homogeneous-heterogeneous switching system was constructed using polystyrene-stabilized PdO nanoparticles (PS-PdONPs) and aryl halide and that tetrabutylammonium bromide (TBAB) would act as a stabilizer for leached palladium species [28]. To evaluate the applicability of the homogeneous-heterogeneous catalytic system, the Suzuki coupling reaction of 2-bromothiophene with arylboronic acid was investigated (Table 1). When the coupling reaction of 2-bromothiophene with 4-methylphenylboronic acid was performed in the absence of TBAB, only $14 \%$ of the product was obtained (Entry 1). In contrast, the same reaction took place smoothly in water at $80{ }^{\circ} \mathrm{C}$ for $1 \mathrm{~h}$ in the presence of TBAB to afford 2-(4-methylphenyl)thiophene in a 78\% yield (Entry 2). After washing with water and diethyl ether and subsequent drying, the recovered catalyst was used for the next run under the same conditions. The yield of the reaction did not decrease markedly, even after five consecutive runs (Entries 3-6). When the reaction solution was analyzed by ICP-AES to determine the extent of palladium leaching after the reaction, palladium was not detectable $(<0.1 \mathrm{ppm})$ 
after any of the runs. However, $0.4 \%$ of palladium leached after heating the mixture of PS-PdONPs and 2-bromothiophene under reaction conditions. A slight increase in the size of palladium was observed by TEM after the recycling experiments (Figure 1). The 1s peak of $\mathrm{N}$ was not observed by XPS analysis, indicating the absence of tetrabutylammonium species in the recovered catalyst (Figure 2). These results suggest that leached palladium species are participating in the catalytic process and are redeposited onto linear polystyrene after the reaction is complete. Indeed, similar processes have been observed in organic solvent $[29,30]$. After the reaction, leached palladium species were re-stabilized onto linear polystyrene, probably due to the chelate effect [31]. The reaction in the presence of SDS gave a low yield (Entry 7) and no corresponding product was obtained in the case of 5-bromo-2-thiophenecarboxylic acid, suggesting that TBAB did not simply act as a phase-transfer catalyst (Entry 8). Both electron-rich and electron-deficient arylboronic acids were reactive, affording the desired coupling products in good yields (Entries 9 and 10). The Suzuki coupling reaction with 1-naphtylboronic acid and 2-thienylboronic acid gave the corresponding coupling products in 87 and 99\% yields, respectively (Entries 11 and 12 ).

Table 1. Suzuki coupling reaction of thienyl halides with arylboronic acids ${ }^{\text {a }}$.

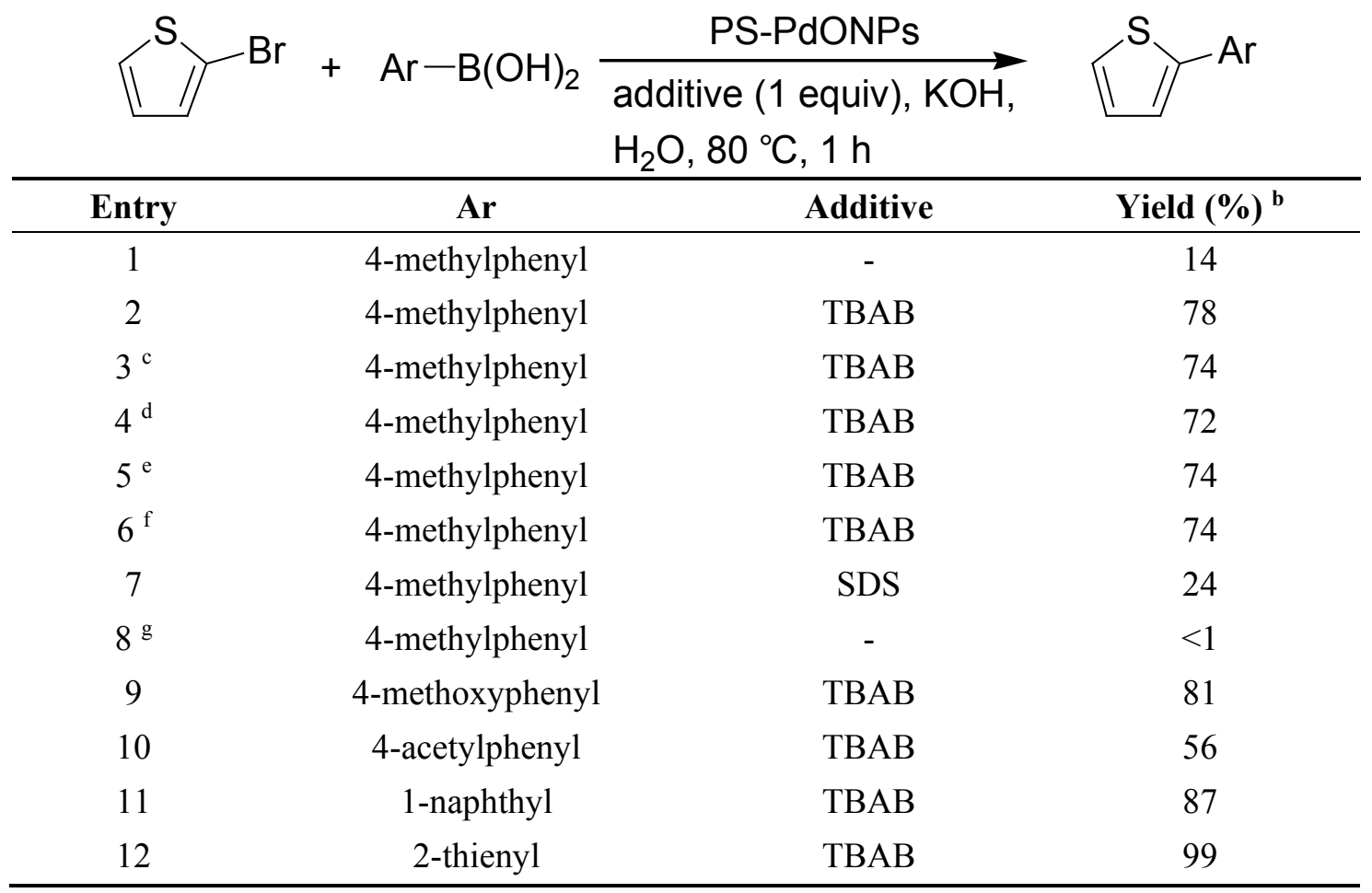

${ }^{a}$ All reactions were performed with 2-bromothiophene $(0.5 \mathrm{mmol})$, arylboronic acid $(0.75 \mathrm{mmol})$, PS-PdONPs (1.5 mol\% of Pd), $1.5 \mathrm{~mol} / \mathrm{L} \mathrm{KOH}$ aqueous solution $(1 \mathrm{~mL}) .{ }^{\mathrm{b}} \mathrm{NMR}$ yields. ${ }^{\mathrm{c}} 2 \mathrm{nd}$ use. ${ }^{\mathrm{d}} 3 \mathrm{rd}$ use. ${ }^{\mathrm{e}} 4$ th use. $^{\mathrm{f}} 5$ th use. $^{\mathrm{g}}$ 5-Bromo-2-thiophenecarboxylic acid was used as a substrate. 

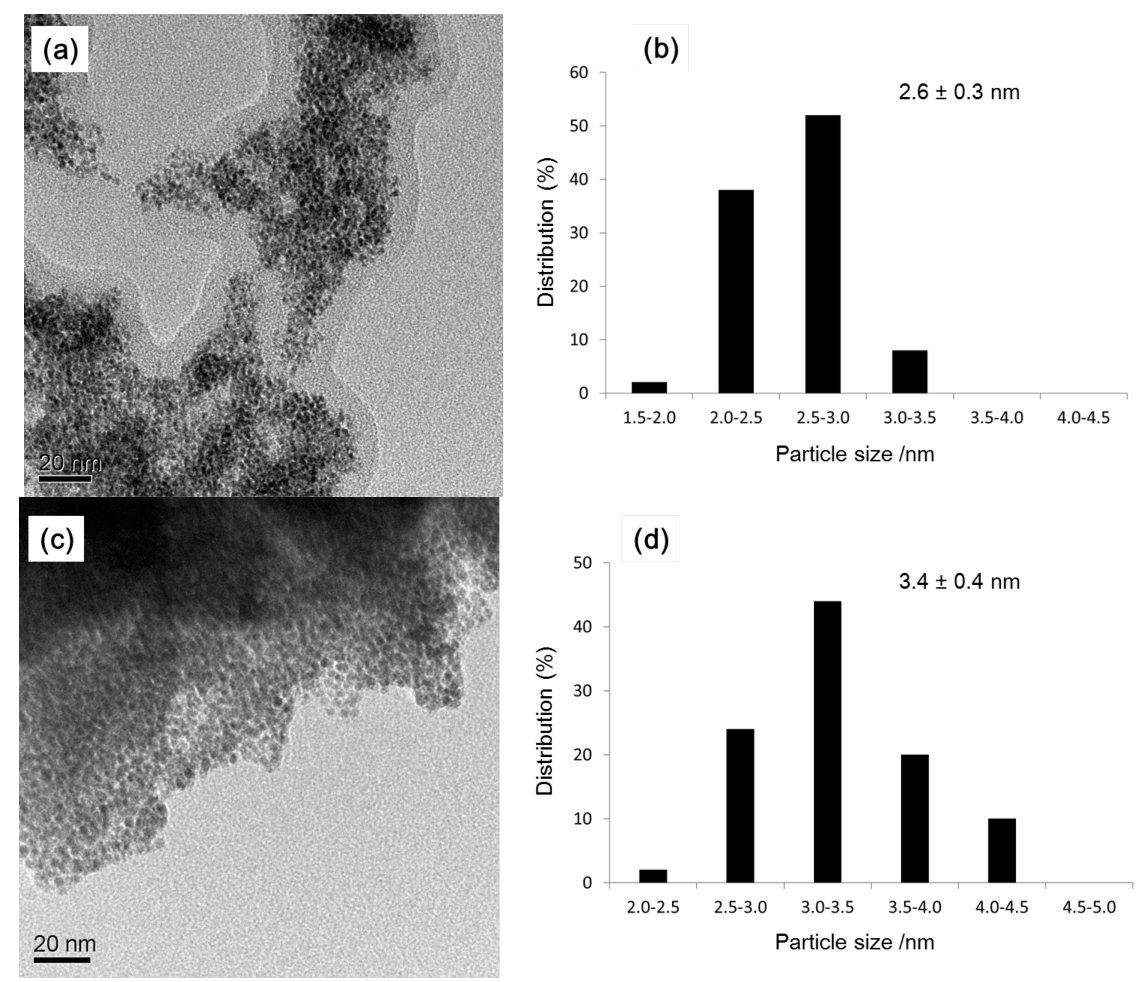

Figure 1. (a) TEM micrograph of PS-PdONPs (scale bar = $20 \mathrm{~nm}$ ); (b) size distribution of PS-PdONPs; (c) TEM micrograph of recovered catalyst after fifth run (scale bar $=20 \mathrm{~nm}$ ); (d) size distribution of recovered catalyst after fifth run.

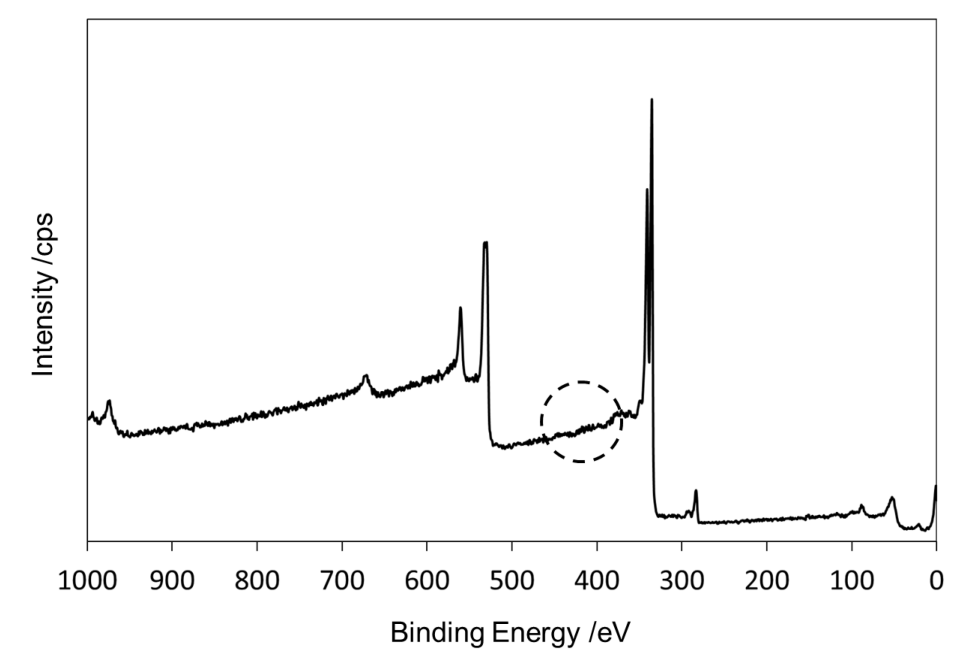

Figure 2. XPS spectrum of the recovered catalyst.

As we reported earlier [32], PS-PdONPs showed low catalytic activity for the Heck reaction with styrene or bromobenzene. Therefore, we also examined the applicability of our boomerang system in the Heck reaction. When the Heck reaction of iodobenzene with styrene was performed in $1.5 \mathrm{~mol} \cdot \mathrm{L}^{-1} \mathrm{KOH}$ aqueous solution at $90{ }^{\circ} \mathrm{C}$ in the absence or presence of TBAB, only $14 \%$ or $17 \%$ of the product was obtained, respectively (Table 2, Entries 1 and 2). However, on using polystyrene-stabilized Pd nanoparticles (PS-PdNPs) as a catalyst, the coupling reaction proceeded efficiently (Entry 4). Based on the mechanism of the Heck reaction using Pd(II) species as the catalyst, the first step is the reduction of 
$\operatorname{Pd}(\mathrm{II})$ to $\operatorname{Pd}(0)$ with alkene. The difference in activity is thought to be caused by the rate of the reduction step (insertion of alkene). Indeed, the reduction of palladium was observed by XPS after treatment of PS-PdONPs with acrylic acid in $1.5 \mathrm{~mol} \cdot \mathrm{L}^{-1} \mathrm{KOH}$ aqueous solution at $90{ }^{\circ} \mathrm{C}$ for $5 \mathrm{~h}$. In contrast, no formation of $\operatorname{Pd}(0)$ was observed after treatment of PS-PdONPs with styrene (Figure 3 ). When the recyclability of the catalyst was examined under the same conditions, catalytic deactivation was observed in the second and third runs. However, similar yields of products were obtained after the third run as in the Suzuki coupling reaction of aryl chloride with arylboronic acid [28]. When the reaction solution was checked by ICP-AES, no palladium species was observed in any runs. In addition, the size of palladium nanoparticles in the recovered catalyst was slightly larger than that of PS-PdNPs (fresh catalyst, Figure 4). The Heck reaction using both electron-rich and electron-deficient aryl iodides also occurred to produce the desired product in good yields. On the other hand, coupling products were obtained in moderate yields from the reaction with 4-methoxystyrene (Entries 18-20).

Table 2. Heck reaction of aryl iodide with styrene ${ }^{\text {a }}$.

\begin{tabular}{|c|c|c|c|}
\hline Entry & $\mathbf{R}_{1}$ & $\mathbf{R}_{2}$ & Yield $(\%)^{b}$ \\
\hline $1^{c, d}$ & $\mathrm{H}$ & $\mathrm{H}$ & 14 \\
\hline $2^{c}$ & $\mathrm{H}$ & $\mathrm{H}$ & 17 \\
\hline $3^{d}$ & $\mathrm{H}$ & $\mathrm{H}$ & 35 \\
\hline 4 & $\mathrm{H}$ & $\mathrm{H}$ & 95 \\
\hline $5^{e}$ & $\mathrm{H}$ & $\mathrm{H}$ & 88 \\
\hline $6^{\mathrm{f}}$ & $\mathrm{H}$ & $\mathrm{H}$ & 56 \\
\hline $7^{\mathrm{g}}$ & $\mathrm{H}$ & $\mathrm{H}$ & 52 \\
\hline $8^{h}$ & $\mathrm{H}$ & $\mathrm{H}$ & 53 \\
\hline $9^{i}$ & $\mathrm{H}$ & $\mathrm{H}$ & 20 \\
\hline $10^{\mathrm{j}}$ & $\mathrm{H}$ & $\mathrm{H}$ & 11 \\
\hline 11 & $4-\mathrm{CH}_{3}-$ & $\mathrm{H}$ & 95 \\
\hline 12 & 4- $\mathrm{CH}_{3} \mathrm{O}-$ & $\mathrm{H}$ & 95 \\
\hline 13 & $4-\mathrm{CF}_{3^{-}}$ & $\mathrm{H}$ & 83 \\
\hline 14 & $2-\mathrm{CH}_{3}-$ & $\mathrm{H}$ & 98 \\
\hline 15 & $\mathrm{H}$ & $\mathrm{Cl}$ & 99 \\
\hline 16 & $4-\mathrm{CH}_{3}-$ & $\mathrm{Cl}$ & 99 \\
\hline 17 & 4- $\mathrm{CH}_{3} \mathrm{O}-$ & $\mathrm{Cl}$ & 96 \\
\hline 18 & $\mathrm{H}$ & $\mathrm{OMe}$ & 74 \\
\hline $\begin{array}{l}19 \\
20\end{array}$ & $\begin{array}{r}4-\mathrm{CH}_{3}- \\
4-\mathrm{CH}_{3} \mathrm{O}-\end{array}$ & $\begin{array}{l}\mathrm{OMe} \\
\mathrm{OMe}\end{array}$ & $\begin{array}{l}77 \\
41\end{array}$ \\
\hline
\end{tabular}

${ }^{a}$ All reactions were performed with aryl iodide $(0.5 \mathrm{mmol})$, styrene $(0.75 \mathrm{mmol})$, catalyst $(1.5 \mathrm{~mol} \%$ of $\mathrm{Pd})$, $1.5 \mathrm{~mol} / \mathrm{L} \mathrm{KOH}$ aqueous solution $(1 \mathrm{~mL}) .{ }^{\mathrm{b}} \mathrm{NMR}$ yield. ${ }^{\mathrm{c}}$ PS-PdONPs was used as a catalyst. ${ }^{\mathrm{d}}$ Reaction was performed in the absence of TBAB for $20 \mathrm{~h}$. e 2nd run. $\mathrm{f} 3 \mathrm{rd}$ run. $\mathrm{g}$ 4th run. ${ }^{\mathrm{h}} 5$ th run. ${ }^{\mathrm{I}} \mathrm{SDS}$ was used as an additive. ${ }^{\mathrm{j}}$ 2-Iodophenol was used as a substrate. 


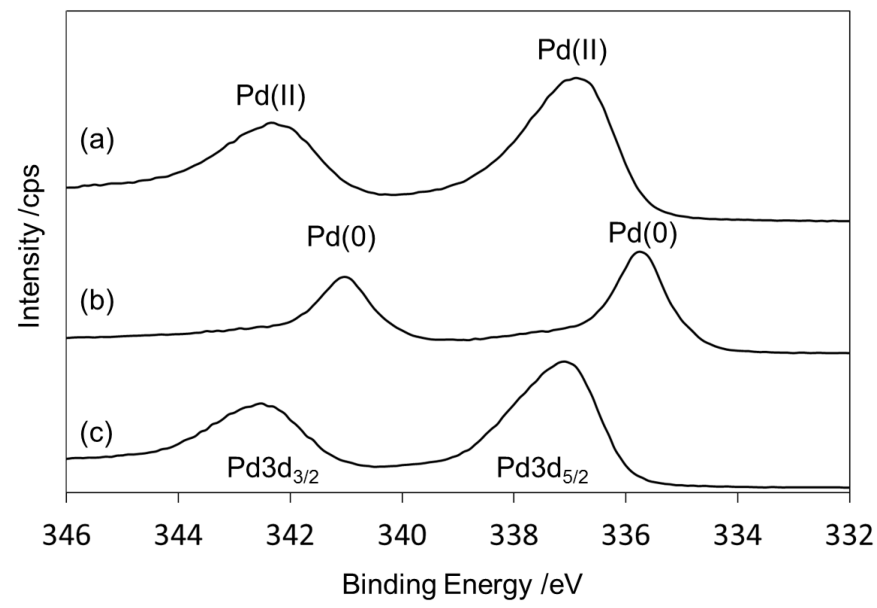

Figure 3. XPS spectrum of the Pd 3d region of the catalyst: (a) PS-PdONPs; (b) after treatment with acrylic acid; (c) after treatment with styrene.

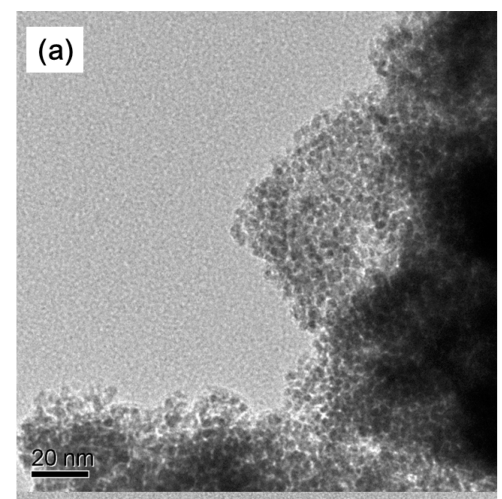

(c)

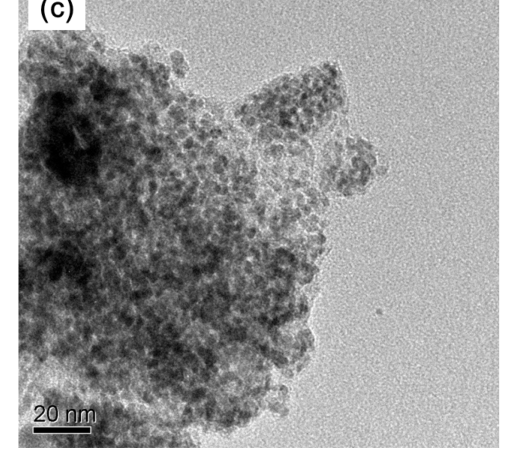

(b)

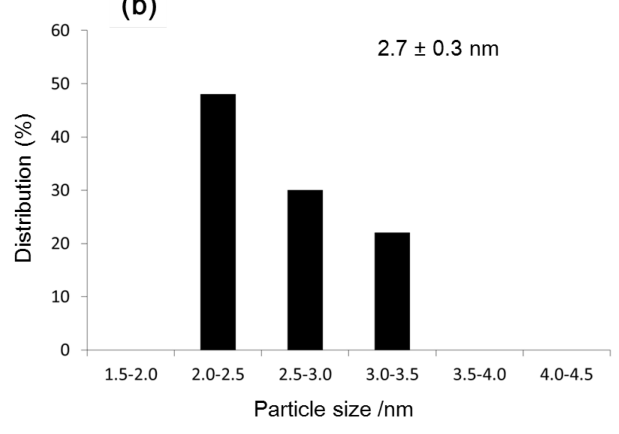

(d)

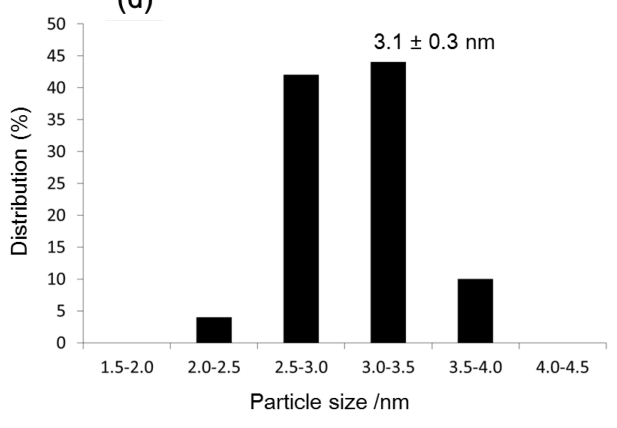

Figure 4. (a) TEM micrograph of PS-PdNPs (scale bar = $20 \mathrm{~nm}$ ); (b) size distribution of PS-PdNPs; (c) TEM micrograph of recovered catalyst after fifth run (scale bar $=20 \mathrm{~nm}$ ); (d) size distribution of recovered catalyst after fifth run.

When the Heck reaction of bromobenzene with acrylic acid was performed in $1.5 \mathrm{~mol} \cdot \mathrm{L}^{-1} \mathrm{KOH}$ aqueous solution at $90{ }^{\circ} \mathrm{C}$ for $5 \mathrm{~h}$, only $5 \%$ of the product was obtained in the absence of additive (Table 3, Entry 1). In contrast, in the presence of TBAB, as expected, an increased product yield was observed (Entry 2). Although a similar yield was obtained with the recovered catalyst, a broader size distribution was observed by TEM in the recovered catalyst (Figure 5). Both electron-rich and electron-deficient aryl bromides were reactive, affording the desired coupling products in high yields (Entries 3-6). 2-Bromotoluene and 1-bromonaphthalene also underwent the Heck reaction with acrylic 
acid under similar conditions to afford 2-methylcinnamic acid and 3-(1-naphthyl)propanoic acid in 78\% and $96 \%$ yields, respectively (Entries 7 and 8 ).

Table 3. Heck reaction of aryl bromide with acrylic acid ${ }^{\text {a }}$.

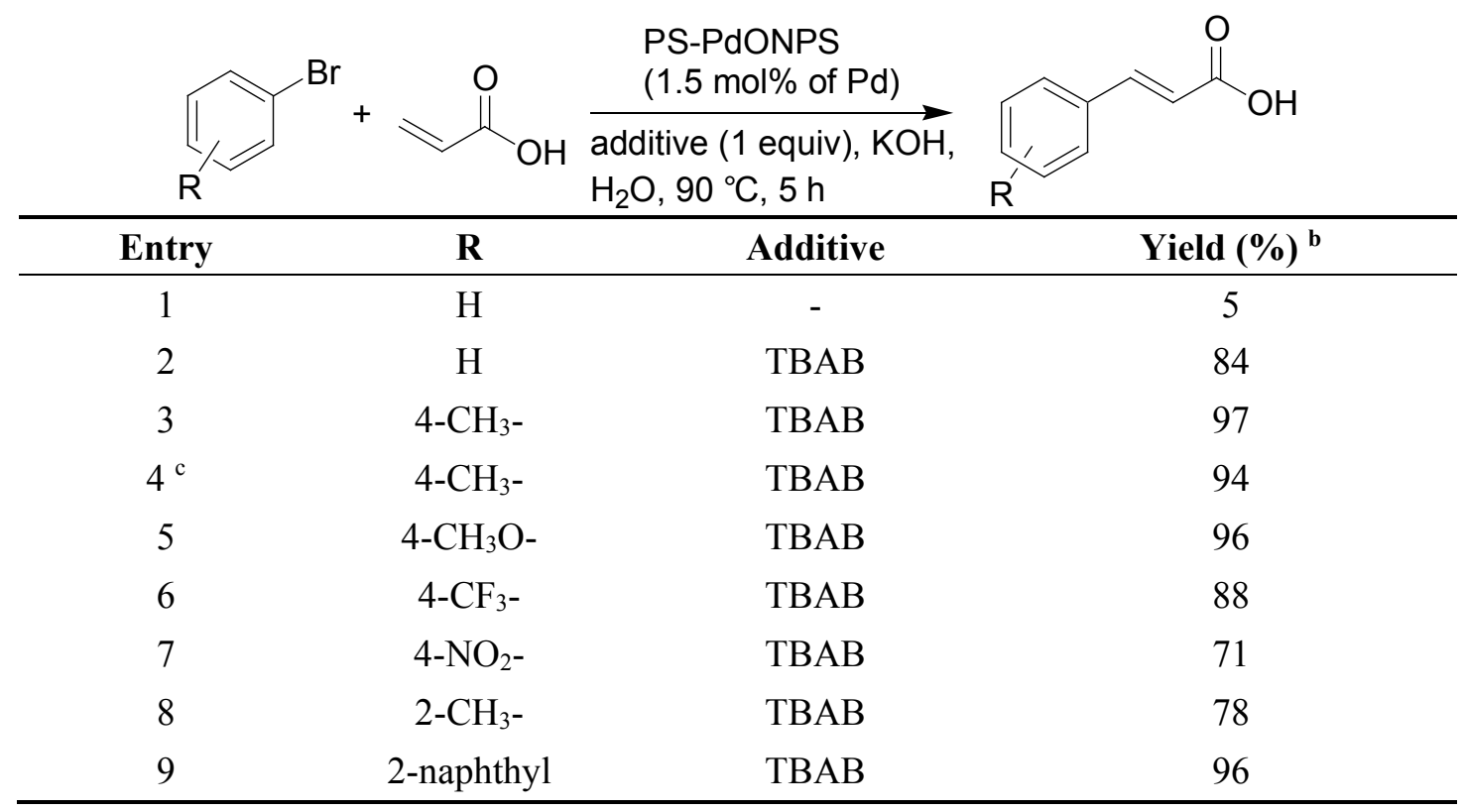

a All reactions were performed with aryl bromide $(0.5 \mathrm{mmol})$, acrylic acid $(0.75 \mathrm{mmol})$, PS-PdONPs (1.5 mol\% of Pd), $1.5 \mathrm{~mol} / \mathrm{L} \mathrm{KOH}$ aqueous solution $(1 \mathrm{~mL}) .{ }^{\mathrm{b}} \mathrm{NMR}$ yield. ${ }^{\mathrm{c}} 2 \mathrm{nd}$ run.
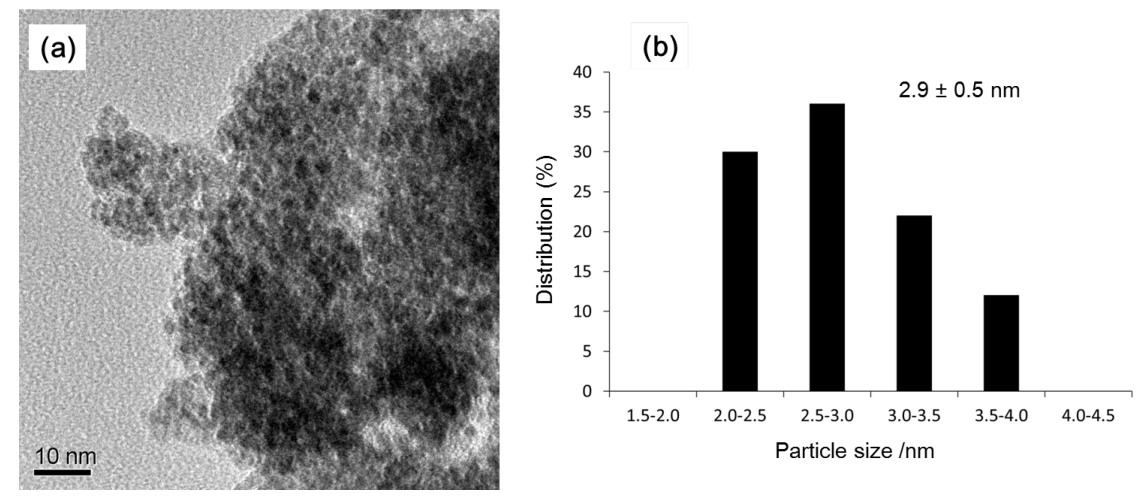

Figure 5. (a) TEM micrograph of the recovered catalyst (scale bar $=10 \mathrm{~nm}$ ); (b) size distribution of the recovered catalyst. 


\section{Experimental Section}

\subsection{Preparation of PS-PdONPs}

To a screw-capped vial with a stirring bar were added $9.0 \mathrm{mg}$ of polystyrene $(85 \mu \mathrm{mol}$ of styrene unit), $\mathrm{Pd}(\mathrm{OAc})_{2} 5.5 \mathrm{mg}(25 \mu \mathrm{mol})$ and $1.5 \mathrm{M}$ aqueous $\mathrm{K}_{2} \mathrm{CO}_{3}$ solution $(3 \mathrm{~mL})$. After stirring at $90{ }^{\circ} \mathrm{C}$ for $1 \mathrm{~h}$, the reaction mixture was filtered with hot water. Subsequently, the polystyrene-stabilized PdO nanoparticles were washed with water $(5 \times 1.0 \mathrm{~mL})$ and acetone $(5 \times 1.0 \mathrm{~mL})$.

\subsection{Preparation of PS-PdNPS}

To a screw-capped vial with a stirring bar were added $1.8 \mathrm{mg}$ of polystyrene (17 $\mu \mathrm{mol}$ of styrene unit), $\mathrm{Pd}(\mathrm{OAc}) 21.0 \mathrm{mg}(5 \mu \mathrm{mol})$, phenylboronic acid $9.1 \mathrm{mg}(0.75 \mathrm{mmol})$ and water $(1 \mathrm{~mL})$. After stirring at $90{ }^{\circ} \mathrm{C}$ for $1 \mathrm{~h}$, the aqueous solution was decanted. Subsequently, the polystyrene-stabilized $\mathrm{Pd}$ nanoparticles were washed with water $(5 \times 1.0 \mathrm{~mL})$ and acetone $(5 \times 1.0 \mathrm{~mL})$.

\subsection{Determination of Loading of the Palladium}

PS-PdONPs (2.9 mg) was placed in a screw-capped vial and then $13 \mathrm{M}$ nitric acid $(5 \mathrm{~mL})$ was added. The mixture was heated at $80{ }^{\circ} \mathrm{C}$ to dissolve completely. After cooling to room temperature, the solution was adjusted to $50 \mathrm{~mL}$ by water, and then, the amount of Pd metal was measured by ICP-AES analysis (15.3 ppm). The amount of Pd in PS-PdNPs was 15.5 ppm.

After the catalytic reaction, the aqueous phase was adjusted to $10 \mathrm{~g}$ by nitric acid, and then, the amount of Pd metal was measured by ICP-AES analysis.

\subsection{Typical Procedures for the Suzuki Coupling Reaction}

To a screw-capped vial with a stirring bar were added 2-bromothiophene $(81.5 \mathrm{mg}, 0.5 \mathrm{mmol})$, 4-methylphenylboronic acid (110 mg, $0.75 \mathrm{mmol}$ ), PS-PdONPs (2.9 mg, $1.5 \mathrm{~mol} \%$ of Pd), TBAB (161 mg, $0.5 \mathrm{mmol}$ ) and $1.5 \mathrm{~mol} \cdot \mathrm{L}^{-1}$ aqueous $\mathrm{KOH}$ solution $(1 \mathrm{~mL})$. After stirring at $80^{\circ} \mathrm{C}$ for $1 \mathrm{~h}$, the reaction mixture was cooled to room temperature by immediately immersing the vial in water $\left(\sim 20^{\circ} \mathrm{C}\right)$ for about $10 \mathrm{~min}$. After separating the catalyst and the aqueous phase by centrifugation, the aqueous phase was decanted. Recovered catalyst was washed with $\mathrm{H}_{2} \mathrm{O}(5 \times 3.0 \mathrm{~mL})$ and diethyl ether $(5 \times 3.0 \mathrm{~mL})$, which were then added to the aqueous phase. The aqueous phase was extracted eight times with diethyl ether. The combined organic extracts were dried over $\mathrm{MgSO}_{4}$ and concentrated under reduced pressure. The product was analyzed by ${ }^{1} \mathrm{H}$ NMR. The recovered catalyst was dried in vacuo and reused. Furthermore, the amount of Pd metal in the aqueous phase determined by ICP-AES analysis was $<0.1 \mathrm{ppm}$.

2-(p-Tolyl)thiophene: ${ }^{1} \mathrm{H}$ NMR $\left(\mathrm{CDCl}_{3}\right) \delta 7.48(\mathrm{~d}, J=8.2 \mathrm{~Hz}, 2 \mathrm{H}), 7.23(\mathrm{~d}, J=3.6 \mathrm{~Hz}, 1 \mathrm{H}), 7.19(\mathrm{~d}$, $J=5.0 \mathrm{~Hz}, 1 \mathrm{H}), 7.15(\mathrm{~d}, J=8.2 \mathrm{~Hz}, 2 \mathrm{H}), 7.06(\mathrm{dd}, J=5.0 \mathrm{~Hz}, 3.6 \mathrm{~Hz}, 1 \mathrm{H}), 2.32(\mathrm{~s}, 3 \mathrm{H}):{ }^{13} \mathrm{C} \mathrm{NMR}$ $\left(\mathrm{CDCl}_{3}\right) \delta 144.6,137.0,131.7,129.5,127.9,125.8,124.2,122.6,21.2$. CAS registry number: 16939-04-1.

2-(4-Methoxyphenyl)thiophene: ${ }^{1} \mathrm{H}$ NMR $\left(\mathrm{CDCl}_{3}\right) \delta 7.55(\mathrm{~d}, J=8.8 \mathrm{~Hz}, 2 \mathrm{H}), 7.20(\mathrm{~m}, 2 \mathrm{H}), 7.05$ $(\mathrm{dd}, J=3.5 \mathrm{~Hz}, J=5.0 \mathrm{~Hz}, 1 \mathrm{H}), 6.92(\mathrm{~d}, J=8.8 \mathrm{~Hz}, 2 \mathrm{H}), 3.83(\mathrm{~s}, 3 \mathrm{H}):{ }^{13} \mathrm{C} \mathrm{NMR}\left(\mathrm{CDCl}_{3}\right) \delta 159.1$, $144.2,127.9,127.2,127.1,123.8,122.0,114.2,55.3$. CAS registry number: 42545-43-7. 
2-(4-Acetylphenyl)thiophene: ${ }^{1} \mathrm{H}$ NMR $\left(\mathrm{CDCl}_{3}\right) \delta 8.02-7.90(\mathrm{~m}, 2 \mathrm{H}), 7.73-7.60(\mathrm{~m}, 2 \mathrm{H}), 7.42(\mathrm{~d}$, $J=3.6 \mathrm{~Hz}, 1 \mathrm{H}), 7.35(\mathrm{~d}, J=5.1 \mathrm{~Hz}, 1 \mathrm{H}), 7.10(\mathrm{dd}, J=5.1 \mathrm{~Hz}, J=3.6 \mathrm{~Hz}, 1 \mathrm{H}), 2.60(\mathrm{~s}, 3 \mathrm{H}):{ }^{13} \mathrm{C} \mathrm{NMR}$ $\left(\mathrm{CDCl}_{3}\right) \delta 197.2,143.0,138.7,135.5,129.1,128.3,126.5,125.5,124.6,26.7$. CAS registry number: 35294-37-2.

2-(o-Tolyl)thiophene: ${ }^{1} \mathrm{H}$ NMR $\left(\mathrm{CDCl}_{3}\right) \delta 7.42(\mathrm{~d}, J=7.2 \mathrm{~Hz}, 1 \mathrm{H}), 7.35(\mathrm{~d}, J=5.0 \mathrm{~Hz}, 1 \mathrm{H})$, $7.20-7.30(\mathrm{~m}, 3 \mathrm{H}), 7.10(\mathrm{dd}, J=5.0 \mathrm{~Hz}, J=3.5 \mathrm{~Hz}, 1 \mathrm{H}), 7.07(\mathrm{~d}, J=3.5 \mathrm{~Hz}, 1 \mathrm{H}), 2.44(\mathrm{~s}, 3 \mathrm{H})$ : ${ }^{13} \mathrm{C} \mathrm{NMR}\left(\mathrm{CDCl}_{3}\right) \delta 143.0,136.1,134.1,130.7,130.4,127.8,127.0,126.5,125.9,125.0,21.5$. CAS registry number: $99846-56-7$.

2-(1-Naphthyl)thiophene: ${ }^{1} \mathrm{H}$ NMR $\left(\mathrm{CDCl}_{3}\right) \delta 8.20(\mathrm{~m}, 1 \mathrm{H}), 7.88(\mathrm{~m}, 1 \mathrm{H}), 7.85(\mathrm{~d}, J=8.4 \mathrm{~Hz}$, $1 \mathrm{H}), 7.56(\mathrm{~d}, J=7.2 \mathrm{~Hz}, 1 \mathrm{H}), 7.55-7.48(\mathrm{~m}, 3 \mathrm{H}), 7.40(\mathrm{~d}, J=5.2 \mathrm{~Hz}, 1 \mathrm{H}), 7.23(\mathrm{~d}, J=3.3 \mathrm{~Hz}, 1 \mathrm{H})$, $7.18(\mathrm{dd}, J=5.2 \mathrm{~Hz}, J=3.3 \mathrm{~Hz}, 1 \mathrm{H}):{ }^{13} \mathrm{C} \mathrm{NMR}\left(\mathrm{CDCl}_{3}\right) \delta 141.7,133.8,132.4,131.8,128.4,128.3$, $128.2,127.3,127.2,126.4,126.0,125.7,125.6,125.2$. CAS registry number: 4632-51-3.

2,2'-Bithiophene: ${ }^{1} \mathrm{H}$ NMR $\left(\mathrm{CDCl}_{3}\right) \delta$ 7.22-7.15 (m, $\left.4 \mathrm{H}\right), 7.05-7.00(\mathrm{~m}, 2 \mathrm{H}):{ }^{13} \mathrm{C} \mathrm{NMR}\left(\mathrm{CDCl}_{3}\right)$ $\delta 137.4,127.5,124.3,123.5$. CAS registry number: 492-97-7.

\subsection{Typical Procedures for the Heck Reaction}

To a screw-capped vial with a stirring bar were added iodobenzene (102 $\mathrm{mg}, 0.5 \mathrm{mmol})$, styrene (78.1 mg, $0.75 \mathrm{mmol}$ ), PS-PdNPs (3.0 mg, $1.5 \mathrm{~mol} \%$ of Pd), TBAB (161 $\mathrm{mg}, 0.5 \mathrm{mmol}$ ) and $1.5 \mathrm{~mol} \cdot \mathrm{L}^{-1}$ aqueous $\mathrm{KOH}$ solution $(1 \mathrm{~mL})$. After stirring at $90{ }^{\circ} \mathrm{C}$ for $15 \mathrm{~h}$, the reaction mixture was cooled to room temperature by immediately immersing the vial in water $\left(\sim 20^{\circ} \mathrm{C}\right)$ for about $10 \mathrm{~min}$. After separating the catalyst and the aqueous phase by centrifugation, the aqueous phase was decanted. Recovered catalyst was washed with $\mathrm{H}_{2} \mathrm{O}(5 \times 3.0 \mathrm{~mL})$ and diethyl ether $(5 \times 3.0 \mathrm{~mL})$, which were then added to the aqueous phase. The aqueous phase was extracted eight times with diethyl ether. The combined organic extracts were dried over $\mathrm{MgSO}_{4}$ and concentrated under reduced pressure. The product was analyzed by ${ }^{1} \mathrm{H}$ NMR. The recovered catalyst was dried in vacuo and reused. Furthermore, the amount of Pd metal in the aqueous phase determined by ICP-AES analysis was $<0.1 \mathrm{ppm}$.

Cinnamic acid: ${ }^{1} \mathrm{H}$ NMR $(\mathrm{CDCl} 3) \delta 7.81(\mathrm{~d}, J=16.0 \mathrm{~Hz}, 1 \mathrm{H}), 7.62-7.50(\mathrm{~m}, 2 \mathrm{H}), 7.42(\mathrm{~m}, 3 \mathrm{H})$, $6.47(\mathrm{~d}, J=16.0 \mathrm{~Hz}, 1 \mathrm{H}):{ }^{13} \mathrm{C} \mathrm{NMR}(\mathrm{CDCl} 3) \delta 172.7,147.0,134.0,130.7,128.9,128.3,117.3$. CAS registry number: $140-10-3$.

4-Methylcinnamic acid: ${ }^{1} \mathrm{H}$ NMR $(\mathrm{CDCl} 3) \delta 7.78(\mathrm{~d}, J=15.9 \mathrm{~Hz}, 1 \mathrm{H}), 7.46(\mathrm{~d}, J=8.1 \mathrm{~Hz}, 2 \mathrm{H}), 7.20$ $(\mathrm{d}, J=8.1 \mathrm{~Hz}, 2 \mathrm{H}), 6.40$ (d, $J=15.9 \mathrm{~Hz}, 1 \mathrm{H}), 2.38$ (s, $3 \mathrm{H}):{ }^{13} \mathrm{C} \mathrm{NMR}(\mathrm{CDCl} 3) \delta 172.6,147.0,141.0$, $131.5,129.8,128.4,116.2,21.4$. CAS registry number: 940-61-4.

4-Methoxycinnamic acid: ${ }^{1} \mathrm{H}$ NMR $(\mathrm{CDCl} 3) \delta 7.75(\mathrm{~d}, J=15.9 \mathrm{~Hz}, 1 \mathrm{H}), 7.52(\mathrm{~d}, J=8.7 \mathrm{~Hz}, 2 \mathrm{H})$, $6.92(\mathrm{~d}, J=8.7 \mathrm{~Hz}, 2 \mathrm{H}), 6.32(\mathrm{~d}, J=15.9 \mathrm{~Hz}, 1 \mathrm{H}), 3.85(\mathrm{~s}, 3 \mathrm{H}):{ }^{13} \mathrm{C} \mathrm{NMR}\left(\mathrm{CDCl}_{3}\right) \delta 172.2,161.8$, $146.5,130.5,126.8,114.4,114.2,55.4$. CAS registry number: 943-89-5.

4-Trifluoromethylcinnamic acid: ${ }^{1} \mathrm{H}$ NMR (DMSO-d 6 ) $\delta 7.90(\mathrm{~d}, J=8.1 \mathrm{~Hz}, 2 \mathrm{H}), 7.74(\mathrm{~d}, J=8.1 \mathrm{~Hz}$, $2 \mathrm{H}), 7.65(\mathrm{~d}, J=15.9 \mathrm{~Hz}, 1 \mathrm{H}), 6.67$ (d, $J=15.9 \mathrm{~Hz}, 1 \mathrm{H}):{ }^{13} \mathrm{C}$ NMR (DMSO-d6) $\delta 167.8,142.2,138.2$, $130.7(\mathrm{q}, J=25.4 \mathrm{~Hz}), 129.7,126.6(\mathrm{q}, J=3.1 \mathrm{~Hz}), 126.0,125.8,122.1$. CAS registry number: 16642-92-5. 
4-Nitrocinnamic acid: ${ }^{1} \mathrm{H}$ NMR (DMSO-d6) $\delta 8.22(\mathrm{~d}, J=8.1 \mathrm{~Hz}, 2 \mathrm{H}), 7.97(\mathrm{~d}, J=8.1 \mathrm{~Hz}, 2 \mathrm{H}), 7.68$ $(\mathrm{d}, J=15.9 \mathrm{~Hz}, 1 \mathrm{H}), 6.74$ (d, $J=15.9 \mathrm{~Hz}, 1 \mathrm{H}):{ }^{13} \mathrm{C} \mathrm{NMR}(\mathrm{CDCl} 3) \delta 167.7,148.4,141.8,141.3,129.7$, 124.4, 123.8. CAS registry number: 619-89-6.

2-Methylcinnamic acid: ${ }^{1} \mathrm{H}$ NMR $(\mathrm{CDCl} 3) \delta 8.00(\mathrm{~d}, J=15.6 \mathrm{~Hz}, 1 \mathrm{H}), 7.60(\mathrm{~d}, J=7.8 \mathrm{~Hz}, 1 \mathrm{H})$, 7.30-7.20 (m, $3 \mathrm{H}), 6.36$ (d, $J=15.6 \mathrm{~Hz}, 1 \mathrm{H}), 2.40$ (s, $3 \mathrm{H}):{ }^{13} \mathrm{C} \mathrm{NMR}\left(\mathrm{CDCl}_{3}\right) \delta 170.4,144.2,138.0$, 133.8, 131.8, 131.2, 126.8, 118.8, 19.7. CAS registry number: 2373-76-4.

Stilbene: ${ }^{1} \mathrm{H}$ NMR (CDCl3) $\delta 7.60(\mathrm{dd}, J=8.4 \mathrm{~Hz}, J=1.5 \mathrm{~Hz}, 4 \mathrm{H}), 7.45$ (tt, $J=7.5 \mathrm{~Hz}, J=1.5 \mathrm{~Hz}$, $4 \mathrm{H}), 7.34(\mathrm{tt}, J=7.2 \mathrm{~Hz}, J=1.5 \mathrm{~Hz}, 2 \mathrm{H}), 7.20$ (s, $2 \mathrm{H}):{ }^{13} \mathrm{C} \mathrm{NMR}\left(\mathrm{CDCl}_{3}\right) \delta 137.4,128.8,128.7,127.7$, 126.6. CAS registry number: 103-30-0.

4-Methylstilbene: ${ }^{1} \mathrm{H}$ NMR $(\mathrm{CDCl} 3) \delta 7.52(\mathrm{~d}, J=7.2 \mathrm{~Hz}, 2 \mathrm{H}), 7.43(\mathrm{~d}, J=8.1 \mathrm{~Hz}, 2 \mathrm{H}), 7.35$ (t, $J=7.5 \mathrm{~Hz}, 2 \mathrm{H}), 7.26(\mathrm{t}, J=7.2 \mathrm{~Hz}, 1 \mathrm{H}), 7.18(\mathrm{~d}, J=8.1 \mathrm{~Hz}, 2 \mathrm{H}), 7.10(\mathrm{~s}, 2 \mathrm{H}), 2.38(\mathrm{~s}, 3 \mathrm{H}):{ }^{13} \mathrm{C} \mathrm{NMR}$ $\left(\mathrm{CDCl}_{3}\right) \delta 137.7,137.5,134.8,129.4,128.7,127.7,127.4,126.5,126.4,21.4$. CAS registry number: 1860-17-9.

4-Methoxystilbene: ${ }^{1} \mathrm{H}$ NMR (CDCl3) $\delta 7.53-7.46$ (m, $\left.4 \mathrm{H}\right), 7.37(\mathrm{t}, J=7.5 \mathrm{~Hz}, 2 \mathrm{H}), 7.25(\mathrm{t}, J=7.2 \mathrm{~Hz}$, $1 \mathrm{H}), 7.10(\mathrm{~d}, J=16.2 \mathrm{~Hz}, 1 \mathrm{H}), 6.94(\mathrm{~d}, J=8.7 \mathrm{~Hz}, 2 \mathrm{H}), 3.85(\mathrm{~s}, 3 \mathrm{H}):{ }^{13} \mathrm{C} \mathrm{NMR}\left(\mathrm{CDCl}_{3}\right) \delta 159.4,137.7$, $130.2,128.5,127.8,127.2,126.4,126.1,114.2,55.3$. CAS registry number: 1694-19-5.

4-Trifluoromethylstilbene: ${ }^{1} \mathrm{H}$ NMR $(\mathrm{CDCl} 3) \delta 7.62-7.56(\mathrm{~m}, 4 \mathrm{H}), 7.54(\mathrm{~d}, J=7.5 \mathrm{~Hz}, 2 \mathrm{H})$, 7.4-7.36 (m, $2 \mathrm{H}), 7.32-7.28(\mathrm{~m}, 1 \mathrm{H}), 7.19(\mathrm{~d}, J=16.5 \mathrm{~Hz}, 1 \mathrm{H}), 7.12(\mathrm{~d}, J=16.5 \mathrm{~Hz}, 1 \mathrm{H}):{ }^{13} \mathrm{C}$ NMR $\left(\mathrm{CDCl}_{3}\right) \delta 140.8,136.6,131.2,129.2(\mathrm{q}, J=32.3 \mathrm{~Hz}), 128.8,128.3,127.1,126.8,126.5,125.6(\mathrm{q}$, $J=3.8 \mathrm{~Hz}), 124.2$. CAS registry number: 1149-56-0.

2-Methylstilbene: ${ }^{1} \mathrm{H}$ NMR $\left(\mathrm{CDCl}_{3}\right) \delta 7.66(\mathrm{~d}, J=7.2 \mathrm{~Hz}, 1 \mathrm{H}), 7.58(\mathrm{~d}, J=7.5 \mathrm{~Hz}, 2 \mathrm{H}), 7.45-7.35$ (m, $3 \mathrm{H}), 7.30-7.25(\mathrm{~m}, 4 \mathrm{H}), 7.05(\mathrm{~d}, J=16.2 \mathrm{~Hz}, 1 \mathrm{H}), 2.48(\mathrm{~s}, 3 \mathrm{H}):{ }^{13} \mathrm{C} \mathrm{NMR}\left(\mathrm{CDCl}_{3}\right) \delta 137.7,136.5$, $135.8,130.5,130.0,128.7,127.5,127.5,126.6,126.3,125.3,20.1$. CAS registry number: 22257-16-5.

4-Chlorostilbene: ${ }^{1} \mathrm{H}$ NMR (CDCl3) $\delta 7.52(\mathrm{~d}, J=7.2 \mathrm{~Hz}, 2 \mathrm{H}), 7.43(\mathrm{~d}, J=8.4 \mathrm{~Hz}, 2 \mathrm{H}), 7.40-7.20$ $(\mathrm{m}, 5 \mathrm{H}), 7.07$ (s, $2 \mathrm{H}):{ }^{13} \mathrm{C} \mathrm{NMR}(\mathrm{CDCl} 3) \delta 137.0,135.9,133.2,129.3,128.8,128.7,127.9,127.7$, 127.4, 126.6. CAS registry number: 1657-50-7.

4-Chloro-4'-methylstilbene: ${ }^{1} \mathrm{H}$ NMR $\left(\mathrm{CDCl}_{3}\right) \delta 7.43(\mathrm{~d}, J=8.1 \mathrm{~Hz}, 2 \mathrm{H}), 7.41(\mathrm{~d}, J=8.4 \mathrm{~Hz}, 2 \mathrm{H})$, $7.31(\mathrm{~d}, J=8.4 \mathrm{~Hz}, 2 \mathrm{H}), 7.17(\mathrm{~d}, J=8.1 \mathrm{~Hz}, 2 \mathrm{H}), 7.07$ (d, $J=15.9 \mathrm{~Hz}, 1 \mathrm{H}), 7.00$ (d, $J=15.9 \mathrm{~Hz}, 1 \mathrm{H})$, $2.36(\mathrm{~s}, 3 \mathrm{H}):{ }^{13} \mathrm{C} \mathrm{NMR}\left(\mathrm{CDCl}_{3}\right) \delta 137.8,136.0,134.1,132.9,129.4,129.2,128.8,127.5,126.4,126.3$, 21.3. CAS registry number: 3041-83-6.

4-Chloro-4'-methoxystilbene: ${ }^{1} \mathrm{H}$ NMR $\left(\mathrm{CDCl}_{3}\right) \delta$ 7.49-7.35 (m, $\left.4 \mathrm{H}\right), 7.33-7.20(\mathrm{~m}, 2 \mathrm{H}), 7.02(\mathrm{~d}$, $J=16.2 \mathrm{~Hz}, 1 \mathrm{H}), 6.95-6.88$ (m, $3 \mathrm{H}), 3.83$ (s, $3 \mathrm{H}):{ }^{13} \mathrm{C} \mathrm{NMR}(\mathrm{CDCl} 3) \delta 159.6,136.2,132.5,129.7$, $128.9,128.8,127.8,127.4,125.3,114.2,55.4$. CAS registry number: 18878-89-2.

4-Methoxy-4'-methylstilbene: ${ }^{1} \mathrm{H}$ NMR $(\mathrm{CDCl} 3) \delta 7.45(\mathrm{~d}, J=8.7 \mathrm{~Hz}, 2 \mathrm{H}), 7.40(\mathrm{~d}, J=8.1 \mathrm{~Hz}$, $2 \mathrm{H}), 7.16(\mathrm{~d}, J=7.8 \mathrm{~Hz}, 2 \mathrm{H}), 7.04(\mathrm{~d}, J=16.2 \mathrm{~Hz}, 1 \mathrm{H}), 6.96(\mathrm{~d}, J=16.2 \mathrm{~Hz}, 1 \mathrm{H}), 6.90(\mathrm{~d}, J=8.7 \mathrm{~Hz}$, $2 \mathrm{H}), 3.83(\mathrm{~s}, 3 \mathrm{H}), 2.36$ (s, $3 \mathrm{H}):{ }^{13} \mathrm{C} \mathrm{NMR}\left(\mathrm{CDCl}_{3}\right) \delta 159.3,137.2,135.0,130.5,129.5,127.7,127.4$, $126.7,126.3,114.3,55.5,21.4$. CAS registry number: $37163-68-1$. 
4,4'-Dimethoxystilbene: ${ }^{1} \mathrm{H}$ NMR (CDCl3) $\delta$ 7.44-7.41 (m, $\left.4 \mathrm{H}\right), 6.94-6.86$ (m, $\left.6 \mathrm{H}\right), 3.83$ (s, $\left.6 \mathrm{H}\right)$ : ${ }^{13} \mathrm{C} \mathrm{NMR}\left(\mathrm{CDCl}_{3}\right) \delta 159.3,130.7,127.7,126.4,114.4,55.6$. CAS registry number: $15638-14-9$.

\section{Conclusions}

In summary, a "boomerang" catalytic system, which allows one to combine the benefits of homogeneous (high catalytic activity) and heterogeneous (easy separation and recycling) catalysts, was constructed by using PS-PdONPs and TBAB. The results with water-soluble substrates or another stabilizer suggested that TBAB did not simply act as a phase-transfer catalyst. A slight increase in the size of palladium was observed by TEM after re-stabilization of the Pd species on linear polystyrene. This system is applicable to the Suzuki coupling reaction of 2-bromothiophene with arylboronic acid and the Heck reaction with aryl styrene or bromide.

\section{Acknowledgements}

The authors are grateful to the Nanomaterials and Microdevices Research Center (NMRC) of OIT for financial and instrumental support.

\section{Author Contributions}

Atsushi Ohtaka and Toshiyuki Okagaki contributed to the experimental design and the article writing and revising. Go Hamasaka, Yasuhiro Uozumi, and Tsutomu Shinagawa contributed to the catalyst characterization. Osamu Shimomura and Ryoki Nomura contributed to a helpful discussion.

\section{Conflicts of Interest}

The authors declare no conflict of interest.

\section{References}

1. Gruttadauria, M.; Giacalone, F.; Noto, R. "Release and Catch" Catalytic Systems. Green Chem. 2013, 15, 2608-2618.

2. Kanaoka, S.; Yagi, N.; Fukuyama, Y.; Aoshima, S.; Tsunoyama, H.; Tsukuda, T.; Sakurai, H. Thermosensitive Gold Nanoclusters Stabilized by Well-Defined Vinyl Ether Star Polymers: Reusable and Durable Catalysts for Aerobic Alcohol Oxidation. J. Am. Chem. Soc. 2007, 129, 12060-12061.

3. Hamamoto, H.; Suzuki, Y.; Yamada, Y.M.A.; Tabata, H.; Takahashi, H.; Ikegami, S. A Recyclable Catalytic System Based on a Temperature-Responsive Catalyst. Angew. Chem. Int. Ed. 2005, 44, 4536-4538.

4. Ohtaka, A.; Tamaki, Y.; Igawa, Y.; Egami, K.; Shimomura, O.; Nomura, R. Polyion Complex Stabilized Palladium Nanoparticles for Suzuki and Heck Reaction in Water. Tetrahedron 2010, $66,5642-5646$.

5. Wittmann, S.; Schätz, A.; Grass, R.N.; Stark, W.J.; Reiser, O. A Recyclable Nanoparticle-Supported Palladium Catalyst for the Hydroxycarbonylation of Aryl Halides in Water. Angew. Chem. Int. Ed. 2010, 49, 1867-1870. 
6. Clavier, H.; Nolan, S.P.; Mauduit, M. Ionic Liquid Anchored "Boomerang" Catalysts Bearing Saturated and Unsaturated NHCs: Recyclability in Biphasic Media for Cross-Metathesis. Organometallics 2008, 27, 2287-2292.

7. Rix, D.; Caïjo, F.; Laurent, I.; Gulajski, L.; Grela, K.; Mauduit, M. Highly Recoverable Pyridinium-Tagged Hoveyda-Grubbs Pre-catalyst for Olefinmetathesis. Design of the Boomerang Ligand Toward the Optimal Compromise Between Activity and Reusability. Chem. Commun. 2007, 3771-3773.

8. Varray, S.; Lazaro, R.; Martinez, J.; Lamaty, F. New Soluble-Polymer Bound Ruthenium Carbene Catalysts: Synthesis, Characterization, and Application to Ring-Closing Metathesis. Organometallics 2003, 22, 2426-2435.

9. Jafarpour, L.; Nolan, S.P. Simply Assembled and Recyclable Polymer-Supported Olefin Metathesis Catalysts. Org. Lett. 2000, 2, 4075-4078.

10. Ahmed, M.; Arnauld, T.; Barrett, A.G.M.; Braddock, D.C.; Procopiou, P.A. Second Generation Recyclable "Boomerang" Polymer Supported Catalysts for Olefien Metathesis: Application of Arduengo Carbene Complexes. Synlett 2000, 1007-1009.

11. Astruc, D. Nanoparticles and Catalysis; Wiley-VCH: Weinheim, Germany, 2008.

12. Yan, N.; Xiao, C.; Kou, Y. Transition Metal Nanoparticle Catalysis in Green Solvents. Coord. Chem. Rev. 2010, 254, 1179-1218.

13. Dell'Anna, M.M.; Mali, M.; Mastrorilli, P.; Rizzuti, A.; Ponzoni, C.; Leonelli, C. Suzuki-Miyaura Coupling under Air in Water Promoted by Polymer Supported Palladium Nanoparticles. J. Mol. Catal. A 2013, 366, 186-194.

14. Beletskaya, I.P.; Kashin, A.N.; Khotina, I.A.; Khokhlov, A.R. Efficient and Recyclabe Catalyst of Palladium Nanoparticles Stabilized by Polymer Micelles Soluble in Water for Suzuki-Miyaura Reaction, Ostwald Ripening Process with Palladium Nanoparticles. Synlett 2008, 1547-1552.

15. Moldal, J.; Modak, A.; Bhaumik, A. One-pot Efficient Heck Coupling in Water Catalyzed by Palladium Nanoparticles Tethered into Mesoporous Organic Polymer. J. Mol. Catal. A 2011, 350, 40-48.

16. Boffi, A.; Cacchi, S.; Ceci, P.; Cirilli, R.; Fabrizi, G.; Prastaro, A.; Niembro, S.; Shafir, A.; Vallribera, A. The Heck Reaction of Allylic Alcohols Catalyzed by Palladium Nanoparticles in Water: Chemoenzymatic Synthesis of $(R)-(-)$-Rhododendrol. ChemCatChem 2011, 3, 347-353.

17. Yu, Y.; Hu, T.; Chen, X.; Xu, K.; Zhang, J.; Huang, J. Pd Nanoparticles on a Porous Ionic Copolymer: A Highly Active and Recyclable Catalyst for Suzuki-Miyaura Reaction under Air in Water. Chem. Commun. 2011, 47, 3592-3594.

18. Zhi, J.; Song, D.; Li, Z.; Lei, X.; Hu, A. Palladium Nanoparticles in Carbon Thin Film-lined SBA-15 Nanoreactors: Efficient Heterogeneous Catalysts for Suzuki-Miyaura Cross Coupling Reaction in Aqueous Media. Chem. Commun. 2011, 47, 10707-10709.

19. Ogasawara, S.; Kato, S. Palladium Nanoparticles Captured in Microporous Polymers: A Tailr-Made Catalyst for Heterogeneous Carbon Cross-Coupling Reactions. J. Am. Chem. Soc. 2010, 132, 4608-4613.

20. Astruc, D.; Lu, F.; Aranzaes, J.R. Nanoparticles as Recyclable Catalysts: The Frontier between Homogeneous and Heterogeneous Catalysis. Angew. Chem. Int. Ed. 2005, 44, 7852-7872. 
21. Gallon, B.J.; Kojima, R.W.; Kaner, R.B.; Diaconescu, P.L. Palladium Nanoparticles Supported on Polyaniline Nanofibers as a Semi-Heterogeneous Catalyst in Water. Angew. Chem. Int. Ed. 2007, 46, 7251-7254.

22. Hu, J.; Wang, Y.; Han, M.; Zhou, Y.; Jiang, X.; Sun, P. A Facile Preparation of Palladium Nanoparticles Supported on Magnetite/s-graphene and Their Catalytic Application in Suzuki-Miyaura Reaction. Catal. Sci. Technol. 2012, 2, 2332-2340.

23. Schätz, A.; Reiser, O.; Stark, W.J. Nanoparticles as Semi-Heterogeneous Catalyst Supports. Chem. Eur. J. 2010, 16, 8950-8967.

24. Fang, P.-P.; Jutand, A.; Tian, Z.-Q.; Amatore, C. Au-Pd Core-Shell Nanoparticles Catalyze Suzuki-Miyaura Reactions in Water through Pd Leaching. Angew. Chem. Int. Ed. 2011, 50, 12184-12188.

25. MacQuarrie, S.; Horton, J.H.; Barnes, J.; McEleney, K.; Loock, H.-P.; Crudden, C.M. Visual Observation of Redistribution and Dissolution of Palladium during the Suzuki-Miyaura Reaction. Angew. Chem. Int. Ed. 2008, 47, 3279-3282.

26. Gniewek, A.; Trzeciak, A.M.; Ziółkowski, J.J.; Kępiński, L.; Wrzyszcz, J.; Tylus, W. Pd-PVP Colloid as Catalyst for Heck and Carbonylation Reactions: TEM and XPS Studies. J. Catal. 2005, 229, 332-343.

27. Ohtaka, A.; Kuroki, R.; Teratani, T.; Shinagawa, T.; Hamasaka, G.; Uozumi, Y.; Shimomura, O.; Nomura, R. Recovery of in situ-generated Pd Nanoparticles with Linear Polystyrene. Green Sus. Chem. 2011, 1, 19-25.

28. Ohtaka, A.; Sakaguchi, E.; Yamaguchi, T.; Hamasaka, G.; Uozumi, Y.; Shimomura, O.; Nomura, R. A Recyclable "Boomerang" Linear Polystyrene-Stabilized Pd Nanoparticles for the Suzuki Coupling Reaction of Aryl Chlorides in Water. ChemCatChem 2013, 5, 2167-2169.

29. Pryjomska-Ray, I.; Gniewek, A.; Trzeciak, A.M.; Ziółkowski, J.J.; Tylus, W. Homogeneous/Heterogeneous Palladium Based Catalytic System for Heck Rection. The Reversible Transfer of Palladium between Solution and Support. Top. Catal. 2006, 40, 173-184.

30. Zhao, F.; Murakami, K.; Shirai, M.; Arai, M. Recyclable Homogeneous/Heterogeneous Catalytic Systems for Heck Reaction through Reversible Transfer of Palladium Species between Solvent and Support. J. Catal. 2000, 194, 479-483.

31. Sun, W.; Zhou, S.; You, B.; Wu, L. Facile Fabrication and High Photoelectric Properties of Hierarchically Ordered Porous $\mathrm{TiO}_{2}$. Chem. Mater. 2012, 24, 3800-3810.

32. Ohtaka, A.; Yamaguchi, T.; Teratani, T.; Shimomura, O.; Nomura, R. Linear Polystyrene-Stabilized PdO Nanoparticle-Catalyzed Mizoroki-Heck Reactions in Water. Molecules 2011, 16, 9067-9076.

(C) 2015 by the authors; licensee MDPI, Basel, Switzerland. This article is an open access article distributed under the terms and conditions of the Creative Commons Attribution license (http://creativecommons.org/licenses/by/4.0/). 